\title{
Forming social systems by coupling minds at different levels of cognition: Design, tools, and research methods
}

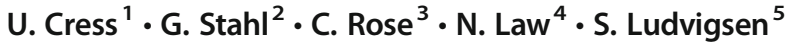 \\ Published online: 4 September 2018 \\ (C) International Society of the Learning Sciences, Inc. 2018
}

Since its inception, the main interest in CSCL has been in describing and supporting the finegrained processes that make collaboration and joint actions happen. CSCL asks how digital tools can be designed in order to support learners' cognition and activities in such a way that they mutually influence each other. How can tools and settings enhance interpersonal exchange and help learners to maximally benefit from each other? How can individual minds be linked in a way that they altogether form a social system?

In ijCSCL, researchers have presented several concepts and theoretical frameworks that aim at describing this synergetic process in more detail, e.g. the concept of conceptual convergence (Roschelle 1992), the idea of group cognition (Stahl 2016), the co-evolution model of individuals and social systems (Cress and Kimmerle 2008), the description of socially shared regulation of learning (Järvelä et al. (2016), or the role that visual representations play in collaborative and interactive meaning making (Furberg et al. 2013; Suthers and Hundhausen 2003). All of this work aims at describing and explaining the complex process of coupling individual minds.

\section{U. Cress \\ u.cress@iwm-tuebingen.de \\ G. Stahl \\ Gerry@ijCSCL.org \\ C. Rose \\ cprose@cs.cmu.edu \\ N. Law \\ nlaw@hku.hk \\ S. Ludvigsen \\ s.r.ludvigsen@iped.uio.no}

1 Leibniz-Institut für Wissensmedien (Knowledge Media Research Center), Tübingen, Germany

2 Drexel University, Philadelphia, PA, USA

3 Carnegie Mellon University Language Technologies Institute and HCI Institute, Pittsburgh, PA, USA

4 University of Hong Kong, Hong Kong, China

5 University of Oslo, Oslo, Norway 
With regard to this coupling process, CSCL research delivers three strands of research topics:

- First of all, CSCL describes the processes for coupling minds: Here, CSCL asks on a more conceptual and theoretical basis how tasks can be designed and learning situations can be orchestrated in ways that lead to joint and co-regulated activities and collaboration. In a nutshell: Which processes facilitate the coupling of individual minds in order to enable shared and collective processes?

- Based on these theoretical considerations, a concrete aim of CSCL is to design tools for coupling minds. Based on its theories and frameworks, CSCL has been developing a variety of tools for collaborative learning. Such tools can be full learning environments like WISE or Knowledge Forum $₫$, but also elements like visualizations, scripts, or prompts. CSCL develops these tools and also aims at providing empirical evidence for their efficacy.

- In order to empirically test the efficacy of CSCL tools, the third interest of CSCL lies in using and establishing methods of analysing the process and outcome of coupling: CSCL develops and makes use of adequate methods for capturing data of interacting individuals, and it aims at analysing relevant data on individual and group levels (Cress 2008).

The coupling processes themselves can take place at different stages of information processing:

- At the early stage of attention, where tools and tasks serve to direct the attention of individuals. This could even be at an unconscious level.

- At the core of the information processing, where the central cognitive processes of thinking and problem solving take place.

- At the metacognitive level where planning and co-regulation happen.

- And - last but not least - at the level of activities. They may be individual or social.

Research focusing on these different stages needs different tools and methods of analysis. In this issue of ijCSCL we present four papers, each of which addresses one of these four stages of collaborative information processing: The paper of Schneider and colleagues uses eyetracking methods and focuses on joint visual attention, the earliest stages of information processing. With its focus on argumentation thinking and the integration of knowledge, the paper of Matuk and Linn addresses the core level of information processing: cognition and thinking. The paper of Hadwin, Bakhjtiar, and Miller deals with meta-cognition, and the paper of Heimbuch, Ollesch, and Bodemer considers writing activities. For addressing these four different levels of coupling minds, each paper makes use of specific theoretical concepts. Each of them designs or uses specific tools that serve to couple minds at a specific level. Finally, each paper applies different methods of data capturing and data analysis as well.

\section{Paper 1: Schneider, Sharma, Cuendet, Zufferey, Dillenbourg, \& Pea: Leveraging mobile eye-trackers to capture joint visual attention in co-located collaborative learning groups}

Joint attention provides a fundamental basis for social interactions. It is so fundamental that collective patterns of visual attention can be observed in babies or animals. So, it is not surprising that joint visual attention is an important basis for social learning. It has been shown that it is also 
relevant for knowledge acquisition in complex domains. For instance, Jarodzka et al. (2013) showed that novices can learn from experts if they are forced to follow their gazes. The study of Schneider et al. describes a more spontaneous form of coupling visual attention. It uses a rich colocated collaboration task offering the use of tangibles, speech and gestures. In the so-called TinkerLamp task, dyads of learners interact with a tangible interface. They have to define design principles for optimizing a warehouse layout by moving small-scale shelves and finding an optimal position for the shelves. During the task, a camera detects the location of the shelves and a projector enhances the physical layout with an augmented-reality layer. The authors use mobile eye trackers to measure eye-movements of both learners. Based on this data, they propose the use of a cross-recurrence graph augmented with spatial information about the eye movement of both persons. They use this to detect which warehouse the two students were jointly looking at.

The study shows that this indicator for joint visual interaction correlates with collaboration quality. It additionally finds a negative correlation between dyads' learning gains and an unbalanced level of participation. So, joint visual attention may be high also in highly coordinated - but ultimately ineffective groups - where one person takes on a visual leadership, and the other just follows and behaves like a free-rider. The authors conclude that a balanced level of visual leadership is a precondition for successful cooperation. With a visualization of the augmented cross-recurrence graphs, the authors propose a method to distinguish between dyads with a balanced and with an unbalanced level of participation.

In sum, the paper shows that in a complex collaboration task, the very basic level of information processing - namely visual attention - is linked to collaboration quality. Referring to the concept of joint visual attention, using the TinkerLamp task and applying the cross-recurrence graph as a visualization tool, the authors took a promising and exciting step for "looking into individual's minds" and detecting that coupling processes can be identified at a very basic level. In CSCL we do not have many studies that focus on these fine-grained processes of coupling minds!

\section{Paper 2: Matuk \& Linn: Why and how do middle school students exchange ideas during science inquiry?}

The second paper deals with the core level of information processing: the level of thinking. Much previous research has shown that the coupling of thinking processes needs interpersonal exchange of knowledge. This requires processes of externalization, internationalization and integration for all the people involved in cooperation, as for example described in the knowledge-integration framework (Linn and Eylon 2011).

Consequently, the study of Matuk and Linn investigates how students in a collaborative setting generate, collect, contribute and integrate ideas, and it measures as an outcome variable how this integration impacts the explanations the students ultimately produce.

For a learning and collaboration tool the study uses the idea manager, which is a tool integrated into the Web-based Inquiry Science Environments (WISE, Slotta and Linn 2009). The idea manager offers each learner two baskets for saving ideas regarding a WISE task: one for private use, which offers only individual access and one as a shared tool, where all other learners also have access. A learner can copy ideas from the private to the shared basket and vice versa. So, this tool makes processes of externalization, internationalization, and integration through the development and modification of ideas observable. The study measures the influence of explicit instruction, where students are asked to sort ideas, share at least one of their ideas, and copy at least one of the ideas of others into their own basket. With this 
instruction, the coupling of minds is supported by an explicit rule that prescribes how often learners have to use both baskets at least. As expected, the results show, that there is a relationship between the diversity of students' ideas, the sources of those ideas (i.e., whether they came from the students themselves or from their peers), and the quality of students' scientific explanations. However, the direction of this relationship is interesting and perhaps surprising: Students who collected more ideas that were not already represented in their private idea collections tended to write poorer explanations, whereas students who generated their own redundant ideas tended to write better explanations. This result shows that just getting to know and dealing with the ideas of others is not enough for the coupling of minds. It is the individual's writing and re-formulation of ideas that primarily enhances idea integration. So, this study seems to propose that for the core cognitive level of thinking and integration of ideas, actions are highly relevant. This is also a main issue in CSCL: Cognition and action are not separated. Action forms cognition, and tools that are developed to stimulate actions deepen the processes of thinking and understanding. This is the case for individual minds, but it might be even more necessary for coupled minds.

\section{Paper 3: Hadwin, Bakhtiar, \& Miller: Challenges in online collaboration: Effects of scripting shared task perception}

The third paper deals with another level of information processing: metacognition. This includes processes of planning, monitoring, and reflecting. These processes are important for individual learning, but even more so for collaborative learning. Tools and processes that support team planning and co-regulation of team members are an essential part of many collaborative environments.

One important aspect of collaborative learning is a self-referential one: Collaborating needs negotiating beliefs and perceptions regarding the collaborative goals and plans about how to achieve the task. This is a complex process of co-construction of goals and plans, where metacognition and regulation processes of collaborating individuals need to be exchanged, negotiated, and aligned to achieve shared or joint regulation (Jarvela and Hadwin 2013).

Analogous to the co-construction of knowledge, the co-construction of meta-cognitive knowledge can be supported by scripts. Scripts help the members to focus on the common task, they facilitate externalizing individual perceptions, thoughts, and plans, and they support the exchange and negotiation of these individual cognitions and enable their alignment. The script used in the paper of Hadwin et al. provides questions about the individual perception of task requirements, and about the challenges a learner anticipates during the task. The learners have to provide their answers in a highly standardized way, which makes all answers comparable. They are accessible in a so called "awareness visualization". This visualization shows individual planning perceptions summarized across group members, so each group member gets to know about the perception of the others, and they can reflect on that.

The study showed that without these kinds of visualizations, individuals rated planning as more problematic, and they encountered more challenges. Consequently, they saw a high need for planning strategies and expected them to be more effective than teamwork strategies. This was different for the groups with visualizations. This group reported higher levels of success with their strategies and it expected both planning and teamwork strategies to be equally effective. So, the awareness visualization seemed to be an efficient tool for the coupling of minds with regard to meta-cognition. It did not only induce reflection processes, but also 
influenced activities and outcomes. The authors used pathways analysis to show this differences between the groups. These decision paths provide transition probability measures from (1) encountering the challenge to (2) selecting a specific strategy and (3) enacting the strategy. With this method, they could consider the relevant processes (that range from perception to action) on the individual level as well as on the group level.

\section{Paper 4: Heimbuch, Ollesch, \& Bodemer: Comparing effects of two collaboration scripts on learning activities for wiki-based environments}

The fourth paper explicitly deals with the level of activities: Specifically, it considers activities in collaborative writing. Collaborative writing is a topic that has become more and more relevant through social media, and especially through the widespread use of wikis. Accordingly, the paper deals with a script that is proposed in Wikipedia, the largest and most-used collaborative writing environment we have. This script promotes a high frequency of individual article edits. It encourages content creators to be bold and perform an edit of an existing article or to create an entirely new article. It encompasses guidelines on how to continue if an edit gets reverted, and it suggests accepting at least three revisions before a discussion becomes required. These different steps are not necessarily designed to be followed in an invariant order, although flow chart representations as used in Wikipedia and the like might suggest a specific order of events. The proposed script is practiced by Wikipedians, but is not informed by any theoretical consideration about collaboration. Consequently, Heimbuch et al. propose an alternative script that aims at providing a more collaborative focus: It promotes participants to discuss any planned article edit and revision upfront, before they make any change to a document. In a first step, the editors have to take planned article edits to the corresponding discussion space. In the second step, they have to start a deliberation process where the community can comment on the proposed wiki edits. Only then can the article revision be performed in the third step. So the alternative collaboration script encourages students to take part in the whole negotiation process where people consider different perspectives and then need to integrate them. The results show that the Wikipedia script leads to a lower quality of article, even if the effect was not as large as the authors may have expected.

Looking across the four papers we find not only differences in the level of information processing they consider, but also in the tools they use and the research methods they propose: All four papers were successful in relating the processes on the level of their focus to some output variables, (e.g., individual learning results or the quality of a shared artifact). Summarized across all four studies we find correlations for all four levels of coupling with task performance: Early steps of information processing like visual attention, central steps of knowledge integration, and meta-cognitive processes of co-planning and performing activities - all can support combining individual systems to a social system, and the coupling of minds can be effectively facilitated at all four levels.

Regarding the tools that enable these coupling processes, three of the four papers focus on scripts. The high efficacy of scripts as coupling means is not surprising, as they force the individuals to consider the actions and perspectives of their partners. The provision of visualizations is more implicit. As many other CSCL studies show, this implicit support-just the provision of visualization - can be effective for supporting cooperation by fostering awareness.

When we consider the papers in this issue, it may also be of interest to note that visualizations play not only an important role as tools for coupling, but also as research methods for data analysis. They seem to also be effective awareness tools for researchers. 


\section{References}

Cress, U. (2008). The need for considering multi-level analysis in CSCL research. An appeal for the use of more advanced statistical methods. International Journal of Computer-Supported Collaborative Learning, 3(1), 69-84. https://doi.org/10.1007/s11412-007-9032-2.

Cress, U., \& Kimmerle, J. (2008). A systemic and cognitive view on collaborative knowledge building with wikis. International Journal of Computer-Supported Collaborative Learning, 3(2), 105-122.

Furberg, A., Kluge, A., \& Ludvigsen, S. (2013). International Journal of Computer Supported Learning, 8(1), 41-64. https://doi.org/10.1007/s11412-013-9165-4.

Jarodzka, H., van Gog, T., Dorr, M., Scheiter, K. \& Gerjets, P. (2013). Learning to see: Guiding students' attention via a Model's eye movements fosters learning. Learning and Instruction, 25, 62-70.

Jarvela, S., \& Hadwin, A. F. (2013). New frontiers: Regulating learning in CSCL. Educational Psychologist, $48(1), 25-39$.

Järvelä, S., Kirschner, P. A., Hadwin, A., Järvenoja, H., Malmberg, J., Miller, M., \& Laru, J. (2016). Socially shared regulation of learning in CSCL: Understanding and prompting individual- and group-level shared regulatory activities. International Journal of Computer Supported Collaborative Learning, 11(3), 263-280. https://oi.org/10.1007/s11412-016-9238-2.

Linn, M. C., \& Eylon, B. S. (2011). Science learning and instruction: Taking advantage of technology to promote knowledge integration. Taylor \& Francis Group: Routledge.

Roschelle, J. (1992). Learning by collaborating: Convergent conceptual change. The Journal of the Learning Sciences, 2(3), 235-276.

Slotta, J. D., \& Linn, M. C. (2009). WISE science. New York: Teachers College Press.

Stahl, G. (2016). Constructing dynamic triangles together: The development of mathematical group cognition. Cambridge, UK: Cambridge University Press.

Suthers, D. D., \& Hundhausen, C. D. (2003). An experimental study of the effects of representational guidance on collaborative learning processes. The Journal of the Learning Sciences, 12(2), 183-218. 